A ONE YEAR STUDY OF CORONAVIRUS SD INFECTION IN MICE

L.D. Jankovsky, J.S. Burks, P. Licari, B.L. Devald, and M.C. Kemp

Center for Neurological Diseases/Rocky Mountain Multiple Sclerosis Center, Veterans Administration Medical Center, University of Colorado School of Medicine, Denver, Colorado, U.S.A.

\title{
INTRODUCTION
}

Coronavirus SD was isolated in mouse brain following intracerebral inoculation of autopsy brain material from a multiple sclerosis patient (1). This isolate is serologically related to the human coronavirus $\mathrm{OC}-43$ and the murine coronavirus A59. It is antigenically distant from JHM, the prototype demyelinating strain of mouse hepatitis virus (2). As previously reported, intracerebral (IC) inoculation of SD into mice induces prominent demyelinating lesions in the spinal cord during the first 30 days post inoculation (PI) (3). This paper describes the pathogenesis of SD infection of mice through PI day 365.

\section{Clinical Course of the Disease}

Three hundred and eighty eight 3 to 4 week old, C57Bl/6J, specific pathogen free mice were inoculated IC with $10^{4}$ plaque forming units of plaque purified SD produced in DBT cells (3). An additional 137 control animals received uninfected DBT cell extract in a similar manner. Evidence of central nervous system (CNS) involvement, including extreme hyperexcitability and/or hind limb paresis, was first observed between 4 and 9 days PI in $21.6 \%$ $(84 / 388)$ of the infected mice. All mice were clinically normal at 30 days PI except for growth retardation in a few mice which had previous neurological signs. 
Morbidity and impending mortality influenced selection of animals sacrificed for histopathology and virus studies in the initial study. Therefore, a second group of mice ( 80 infected, 10 control) were inoculated and evaluated to day 365 PI. Of these animals, 25\% (20/80) exhibited neurologic signs in the first 8 days PI. Seven clinically ill mice died. Of the 13 remaining mice with neurological signs, 10 recovered by day $10 \mathrm{PI}$ and 3 continued to exhibit signs. The 3 mice with neurologic signs were sacrificed on day 12. Five of the 10 recovered mice either died or experienced recurrent neurologic signs between day 52 and 146 PI. Six mice which had not demonstrated clinical signs early, developed neurological signs between day 109 and 290 PI. The remainding 35 mice had no neurologic signs at any time before being sacrificed at various points throughout the study.

\section{Virus Studies}

Infectious virus, by plaque assay, was recoverable up to PI day 6 from brain and spinal cord (3). Virus could be detected occasionally by cocultivation between PI days 7 and 12 . Infectious virus was not detectable after day 12 PI by any method. Viral antigen was consistently demonstrated in CNS lesions by indirect immunofluorescence through day 10 and by autoradiography techniques (4) through day 30 PI.

\section{Histopathology}

Fixation and preparation of tissues for light and electron microscopy have previously been described (3). In the initial study $95 \%$ of the infected mice examined had histologic evidence of CNS involvement occurring during the first 30 days PI. In the first week an acute meningoencephalitis was noted. During the second, third and fourth weeks, well circumscribed demyelinated lesions of the spinal cord were the prominent histopathologic feature. Initially, the demyelinated lesions were small, subpial and devoid of inflammatory cells other than macrophages. Large demyelinated lesions extending over several vertebral segments were observed on PI day 12 (Fig. 1). Perivascular demyelination was associated with prominent lymphoid cuffs (lymphocytes, plasma cells and macrophages). Demyelination was not observed by light microscopy beyond PI day 30. However, by electron microscopy, myelin breakdown was observed in the spinal cord of two animals 90 days PI. Remyelinating activity was detected by day 12 PI and was prominent by day 20 PI. Thinly myelinated axons, with oligodendroglial cell cytoplasm interposed between myelin lamellae and axonal membranes, were numerous at the periphery of spinal cord lesions (Fig. 2). 


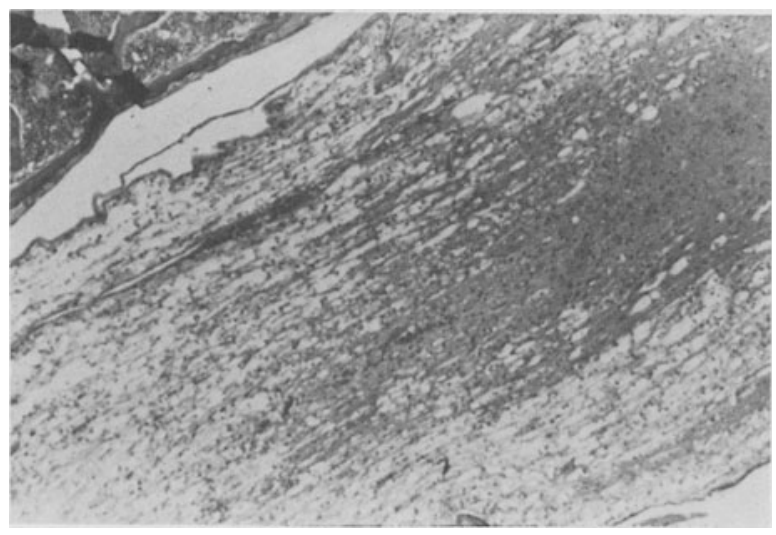

Figure 1: Diffuse Demyelination. In this longitudinal section of mouse spinal cord on day $12 \mathrm{PI}$, diffuse demyelination extends over several vertebral segments (light micrograph 40X).

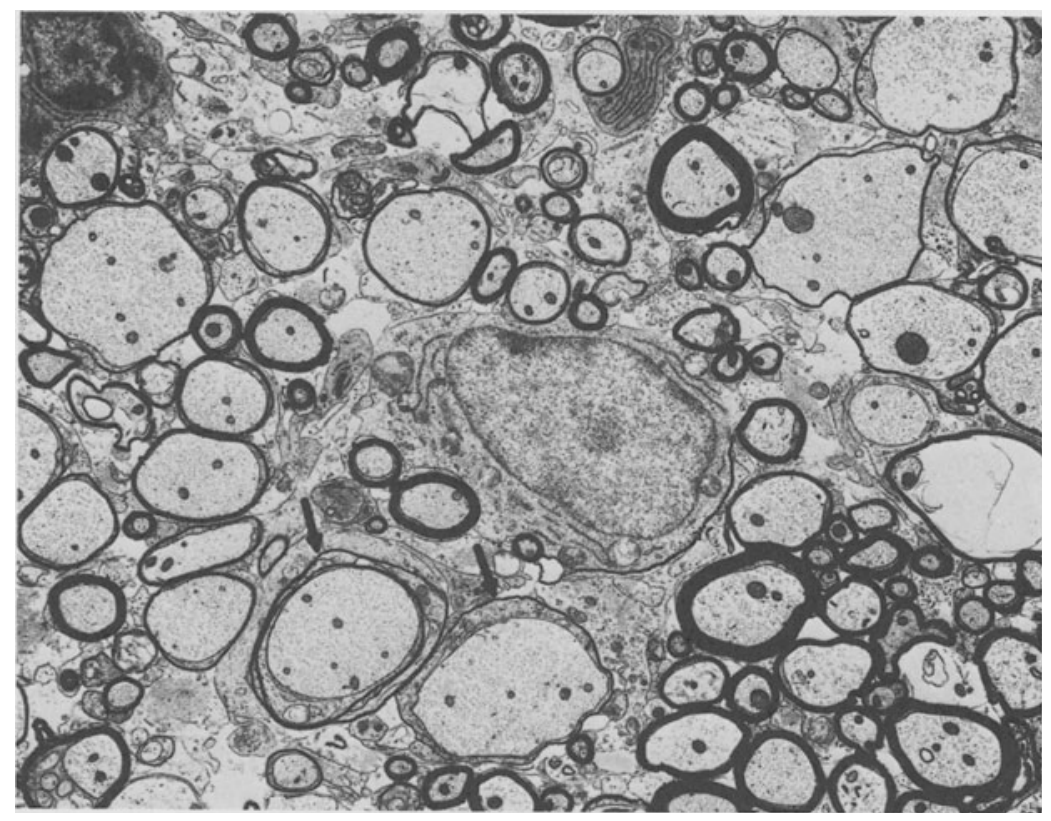

Figure 2: Remyelination. Several thinly myelinated axons surround an oligodendroglial cell in the center of this cross section of mouse spinal cord on day 20 PI. Dark staining oligodendroglial cytoplasm (arrows) is interposed between axonal membranes and myelin lamellae (electron micrograph, 5600X). 
Hydrocephalus was the major neuropathologic feature of SD infection beyond PI day 30 (Fig. 3). Temporal cortices of hydrocephalic mice exhibited a loss of neurons and an abundance of reactive atrocytes. Aqueductal stenosis was not observed in any animal. Of the $35 \mathrm{mice}$ which had no neurologic signs throughout the course of this study, $8(23 \%)$ were found to be hydrocephalic when examined histologically between days 164 and 335 PI. Of the 6 mice which had no neurologic signs early in the study but developed neurologic signs between day 109 and day 290 PI, all had hydrocephalus. Of the 5 mice which had early signs but remained clinically normal after day 10 PI, 2(40\%) had hydrocephalus. Of the 5 mice which had early signs and recovered and later developed a recurrence of neurological signs or were found dead in their cages, only one was examined and it had hydrocephalus.

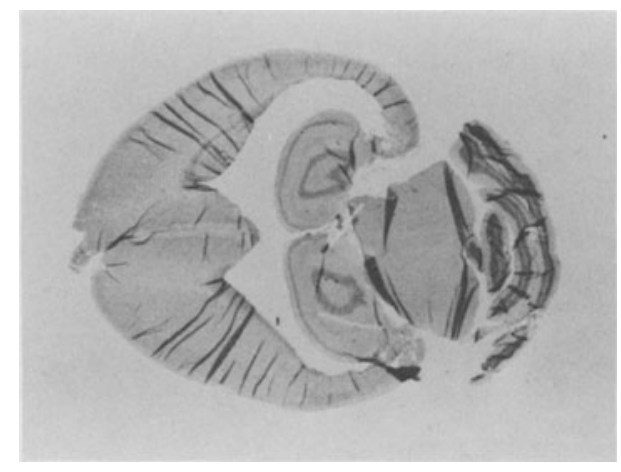

Figure 3: Hydrocephalus. In this horizontal section of mouse brain 109 days PI, temporal cortices are extremely thin and lateral ventrical are markedly dilated (light micrograph, 6.5X).

Immunoglobulin $\mathrm{G}$ was first detected in brain and spinal cord lesions by autoradiography (3) on PI day 6 and remained detectable in lesions for the first 30 days PI. The presence of IgG has not been evaluated beyond day 30 PI.

\section{Comment}

Virus induced demyelination may result directly from viral mediated cellular dysfunction/destruction or indirectly through an immunopathologic process (5). The mechanism of SD induced demyelination is unclear. An immunopathologic process is less likely since treatment with cyclophosphamide does not inhibit demyelination (6). Although infectious virus could only be recovered for the first six days PI, viral antigen persisted and corresponded with the period of active demyelination in the first 30 days PI. Therefore, viral antigenic expression correlated with demyelination, but production of infectious virus did not. 
Hydrocephalus was prominent after 30 days PI in all mice with clinical signs and $23 \%$ of mice which never developed clinical signs. Virus induced hydrocephalus has been reported following obstruction of the aqueduct of Sylvius (7), as a compensatory change secondary to destructive lesions in the cerebral hemispheres and as a function of insufficient reabsorption of the cerebrospinal fluid. Residual destructive changes in temporal cortices and the absence of aqueductal stenosis suggests SD induced hydrocephalus is of a compensatory nature. Late clinical signs associated with hydrocephalus may indicate continuing or intermittent tissue destruction.

Are the mechanisms of demyelination in this SD infection in mice similar or different from demyelination seen in multiple sclerosis? Similarities include: 1) A biphasic disease occurs in some SD infected animals, and a relapsing/remitting course is seen in most MS patients; 2) Axons are spared in a fulminant demyelinating process in both; 3) Lesions are commonly located along surfaces or perivascular areas in both; 4) Prominent lymphocytic perivascular inflammation is noted at certain stages in both; 5) IgG is present in the demyelinating lesions in both and 6) Remyelination occurs at the periphery of the demyelinated lesions in both.

Significant differences between SD infection in mice and MS include: 1) Obvious clinical signs are absent in SD infection in mice during the period of active demyelination between PI days 10 and $30 ; 2$ ) There is a paucity of demyelinating lesions after PI day 30 in SD infected mice; 3) MS is not known to be associated with an encephalitis preceeding demyelination as is observed in SD infection, and 4) some SD infected mice develop marked hydrocephalus.

The relationship between coronavirus SD and multiple sclerosis is uncertain and will remain uncertain until MS brain tissue and control tissue are evaluated for the presence or absence of SD viral genome.

\section{REFERENCES :}

1. Burks, J.S., B.L. DeVald, L.D. Jankovsky, J.C. Gerdes, 1980 . Two Coronaviruses Isolated from Central Nervous System Tissue of Two Multiple Sclerosis Patients. Science. 209:933-934.

2. Gerdes, J.C., I. Klein, B.L. DeVald, J.S. Burks, 1981. Coronavirus Isolates SK and SD from Multiple Sclerosis Patients Are Serologically Related to Murine Coronavirus A59 and JHM and Human Coronavirus OC-43, but not to Human Coronavirus 229E. J. Virol. 38:231-238.

3. Mendelman, P.M., L.D. Jankovsky, R.S. Murray, P. Licari, B. 
DeVald, J.C. Gerdes, J.S. Burks, 1983. Pathogenesis of Coronavirus SD in Mice I. Prominent Demyelination in the Absence of Infectious Virus Production. Arch. of Neurol.

4. J.C. Gerdes, I. McNally, L. Hileman, J.S. Burks, 1982. Autoradiographic Detection of IgG and Viral Antigens. J. Immunol. Meth. 54:191-202.

5. Weiner, L.P., S.A. Stohlman, 1978. Viral Models of Demyelination. Neurology 28:111-114.

6. J.S. Burks, L. Jankovsky, I.T. McNally, M.C. Kemp. 1983. Immunosuppression with Cyclophosphamide Does Not Prevent Demyelination or Result in Uncontrolled Viral Replication in Coronavirus SD Infected Mice. 1983. See these proceedings.

7. Johnson, R.T., K.P. Johnson, C. Jill Edmonds, 1967. Virus Induced Hydrocephalus: Development of Aqueductal Stenosis After Mumps Infection. Science. 157:1066-1067

This study was supported by grants from the Veterans Administration and the Kroc Foundation. We thank Karon Keys for her patience and typing in preparation of this manuscript. 